\title{
REMARKS ON GIESEKER'S DEGENERATION AND ITS NORMALIZATION
}

\author{
Xiaotao Sun
}

\section{INTRODUCTION}

Let $X$ be a smooth projective curve of genus $g \geq 2$ and let $\mathcal{U}_{X}(r, d)$ be the moduli space of stable vector bundles of rank $r$ and degree $d$ on $X$. Assume $(r, d)=1$, then $\mathcal{U}_{X}(r, d)$ is a smooth projective variety of dimension $r^{2}(g-1)+1$. Let $c_{i}\left(\mathcal{U}_{X}(r, d)\right)$ be the Chern classes $c_{i}\left(\Omega_{\mathcal{U}_{X}(r, d)}^{1}\right)$ in the deRham cohomology of $\mathcal{U}_{X}(r, d)$. A conjecture of Newstead and Ramanan states that

$$
c_{i}\left(\mathcal{U}_{X}(2,1)\right)=0 \quad \text { for } i>2(g-1) .
$$

It was proved by Gieseker [Gi] on 1984 by using degeneration. In fact, by degenerating $X$ into an irreducible curve $X_{0}$ with one node, Gieseker constructs a degeneration $\mathcal{G}_{X_{0}}(2,1)$ of $\mathcal{U}_{X}(2,1)$ which has normal crossing singularities. The normalization $\mathcal{N}_{\widetilde{X}_{0}}(2,1)$ of $\mathcal{G}_{X_{0}}(2,1)$, where $\widetilde{X}_{0}$ is the normalization of $X_{0}$, is smooth and the vanishing of $c_{i}\left(\mathcal{U}_{X}(2,1)\right)$ is equivalent to the vanishing of $c_{i}\left(\Omega_{\mathcal{N}_{\widetilde{X}_{0}}(2,1)}(\log D)\right)$, where $D$ is the divisor which maps to the singular locus of $\mathcal{G}_{X_{0}}(2,1)$. Then he shows that $\mathcal{N}_{\widetilde{X}_{0}}(2,1)$ can be obtained, by explicit blowing up and blowing down, from a fibre bundle over $\mathcal{U}_{\widetilde{X}_{0}}(2,1)$ with fibre being the so called wonderful compactification of $G L(2)$, where $\widetilde{X}_{0}$ has genus $g-1$. Thus he is able to prove the vanishing of Chern classes by using induction to genus $g$. Gieseker proposed also in [Gi] to generalize his theory to higher rank.

Gieseker's degeneration $\mathcal{G}_{X_{0}}(2,1)$ consists of pairs $\left(X_{k}, E\right)$ such that the curve $X_{k}$ embedding through $E$ in a Grassmannian is a stable Hilbert point. In rank two case, Gieseker was able to write down all of the types of $\left(X_{k}, E\right)$, which is however difficult to generalize to higher rank. Around 1999, D. S. Nagaraj and C. S. Seshadri made an important observation that one should relate Gieseker's degeneration to moduli spaces of stable torsion free sheaves on $X_{0}$. Let $\pi: X_{k} \rightarrow X_{0}$ be the canonical morphism contractiong the chain of projective lines. They consider in [NS] the pairs $\left(X_{k}, E\right)$ such that $\pi_{*} E$ is a stable sheaf on $X_{0}$ and construct their moduli space as a GIT quotient $\mathcal{G}_{X_{0}}(r, d)$ which has normal crossing singularities. It remains difficult to work out the relationship between $\mathcal{G}_{X_{0}}(r, d)$ and $\mathcal{U}_{\widetilde{X}_{0}}(r, d)$ so that the induction on the genus $g$ is applicable.

In a very recent work [KL], Young-Hoon Kiem and Jun Li provide an alternate construction of the degeneration. They construct the degeneration as an algebraic

The work is supported by the outstanding young grant of NFSC at contract number 10025103 and a RGC grant of Hong Kong at HKU7025/03P. 
space with normal crossing singularities, which parameterizes $\left(X_{k}, E\right)$ such that $E$ is a suitable defined stable bundle on $X_{k}$ (See Definition 1.1 of [KL] where a sufficiently small $\epsilon>0$ is needed). With the cost that the moduli spaces may not be projective varieties, they construct a family of proper separated smooth algebraic spaces $M^{\alpha}(0<\alpha<1)$, which are moduli spaces of certain bundles, so that $M^{1^{-}}$is normalization of the degeneration and $M^{0^{+}}$is a fiber bundle over $\mathcal{U}_{\widetilde{X}_{0}}(r, d)$ with fiber being the wonderful compactification of $G L(r)$ (studied carefully by Ivan Kausz in [K1]). When $\alpha$ moves from $0^{+}$to $1^{-}$, they work out a very precise description of the variation of $M^{\alpha}$ in the case of rank 3. By using these description and the inductive assumption of $\mathcal{U}_{\widetilde{X}_{0}}(3,1)$, they prove that

$$
c_{i}\left(\mathcal{U}_{X}(3,1)\right)=0 \quad \text { for } i>6 g-5 .
$$

Base on the proved vanishing result, we would like to make the following

Conjecture. Let $(r, d)=1$ and $\mathcal{U}_{X}(r, d)$ be the moduli space of stable bundles of rank $r$ and degree $d$ over a smooth projective curve $X$ of genus $g \geq 2$. Then

$$
c_{i}\left(\mathcal{U}_{X}(r, d)\right)=0 \quad \text { for } i>r(r-1)(g-1) .
$$

In trying of understand the work [KL] of Young-Hoon Kiem and Jun Li, we show in this note that their degeneration coincides with the degeneration $\mathcal{G}_{X_{0}}(r, d)$ of Nagaraj and Seshadri. We also construct a family of GIT quotients $M^{\alpha}(0<$ $\alpha<1$ ) which are fine moduli spaces of $\alpha$-stable Gieseker vector bundle data except finite number of $\alpha \in I_{r}$. The moduli space $M^{1^{-}}$is the normalization $\mathcal{N}_{\widetilde{X}_{0}}(r, d)$ of $\mathcal{G}_{X_{0}}(r, d)$ and $M^{0^{+}}$is a fiber bundle over $\mathcal{U}_{\widetilde{X}_{0}}(r, d)$ with fiber being the wonderful compactification of $G L(r)$. The vanishing of Chern classes for $M^{0^{+}}$has been proved very recently by Michel Brion and Ivan Kausz in [BK]. Thus it remains to work out the relationship between $M^{\alpha^{-}}$and $M^{\alpha^{+}}$when $\alpha \in I_{r}$.

Acknowledgement. The work was done during my stay at the university of Hong Kong. I would like to thank Prof. Ngaiming Mok for many helpful discussions.

\section{$\S 1$ Generalized Gieseker moduli spaces}

Let $X_{0}$ be the irreducible curve with one node $x_{0}$, let $\pi: \widetilde{X}_{0} \rightarrow X_{0}$ be the normalization and $\pi^{-1}\left(x_{0}\right)=\left\{x_{1}, x_{2}\right\}$. The semistable models of $X_{0}$, by definition, are semistable curves $X_{0}, X_{k}=\widetilde{X}_{0} \cup R(\mathrm{k}=12 \ldots)$, where $R$ is a chain of $k$ projectives lines and $\widetilde{X}_{0} \cap R=\left\{x_{1}, x_{2}\right\}$. We still use $\pi: X_{k} \rightarrow X_{0}$ to denote all of the morphisms that contract the chain $R$. The generalized Gieseker's moduli spaces are moduli spaces of suitable defined semistable pairs $\left(X_{k}, E\right)$, where $E$ is a vector bundle on $X_{k}$. Two pairs $\left(X_{k}, E\right),\left(X_{k}^{\prime}, E^{\prime}\right)$ are called equivalent if $E \cong \sigma^{*} E^{\prime}$, where $\sigma: X_{k} \rightarrow X_{k}^{\prime}$ is an isomorphism that is identity on $\widetilde{X}_{0}$.

Assume $(r, d)=1$, Nagaraj and Seshadri [NS] prove that there is a canonical structure of projective variety $\mathcal{G}_{X_{0}}(r, d)$ on the set

$$
G(r, d)=\left\{\begin{array}{l}
\text { Equivalence classes of pairs }\left(X_{k}, E\right), \text { where } E \text { is a vector } \\
\text { bundle on } X_{k} \text { of rank } r \text { and degree } d \text { which is strictly } \\
\text { positive on each } \mathbb{P}^{1} \text { of } R \text { and } \pi_{*}(E) \text { is stable on } X_{0} .
\end{array}\right\}
$$


Remark that stability of $\pi_{*}(E)$ implies $k \leq r$. The singularities of $\mathcal{G}_{X_{0}}(r, d)$ are (analytic) normal crossings. Nagaraj and Seshadri construct $\mathcal{G}_{X_{0}}(r, d)$ as a GIT quotient, which we are going to recall briefly.

Fix a line bundle $\mathcal{O}_{X_{0}}(1)=\mathcal{O}_{X_{0}}(x), x \neq x_{0}$. Let $N$ be large enough such that $E(N):=E \otimes \pi^{*} \mathcal{O}_{X_{0}}(N)$ is generated by global sections and $H^{1}(E(N))=0$ for any $\left(X_{k}, E\right) \in G(r, d)$. Let $P(N)=r N+d+r(1-g)$. Then we define the functor

$$
\mathcal{Y}:(\mathbb{C} \text {-schemes }) \rightarrow(\text { Sets }),
$$

$\mathcal{Y}(T):=$ set of closed subschemes $\mathcal{C} \subset X_{0} \times T \times G r(P(N), r)$ such that

(1) the induced projection map $\mathcal{C} \rightarrow T \times G r(P(N), r)$ is a closed embedding over $T$. Let $\mathcal{O}_{\mathcal{C}}^{P(N)} \rightarrow \mathcal{F} \rightarrow 0$ be the pull-back of tautological quotient on $\operatorname{Gr}(P(N), r)$.

(2) the projection $\mathcal{C} \rightarrow T$ is a flat family of curves $\left\{\mathcal{C}_{t}\right\}_{t \in T}$ such that $\mathcal{C}_{t} \cong X_{k}$, and the projection $\mathcal{C} \rightarrow X_{0} \times T$ over $T$ is the canonical morphism $\pi: \mathcal{C} \rightarrow$ $X_{0} \times T$ contracting the chains of projective lines.

(3) the vector bundles $\mathcal{F}_{t}=\left.\mathcal{F}\right|_{\mathcal{C}_{t}}$ on $\mathcal{C}_{t}(t \in T)$ are of rank $r$ and degree $d+r N=P(N)+r(g-1)$. The qoutients $\mathcal{O}_{\mathcal{C}_{t}}^{P(N)} \rightarrow \mathcal{F}_{t}$ induce isomorphisms

$$
H^{0}\left(\mathcal{O}_{\mathcal{C}_{t}}^{P(N)}\right) \cong H^{0}\left(\mathcal{F}_{t}\right)
$$

Proposition 1.1 ([Gi], [NS]). $\mathcal{Y}$ is represented by a $S L(P(N))$-stable subscheme $\mathcal{H} \subset \operatorname{Hilb}^{P_{1}}\left(X_{0} \times G r(P(N), r)\right)$. The $\mathcal{H}$ is irreducible with only normal crossing singularities.

Let $\mathcal{C} \subset X_{0} \times \mathcal{H} \times \operatorname{Gr}(P(N), r), \pi: \mathcal{C} \rightarrow X_{0} \times \mathcal{H}$ and $\mathcal{O}_{\mathcal{C}}^{P(N)} \rightarrow \mathcal{F} \rightarrow 0$ be the universal objects. Let $\mathcal{E}=\mathcal{F} \otimes \pi^{*} \mathcal{O}_{X_{0}}(-N)$ and $\mathbb{C}^{P(N)} \otimes \pi^{*} \mathcal{O}_{X_{0}}(-N) \stackrel{q}{\rightarrow} \mathcal{E} \rightarrow 0$ on $\mathcal{C}$. For $y=\left(\mathbb{C}^{P(N)} \otimes \mathcal{O}_{X_{k}}(-N) \stackrel{q_{y}}{\longrightarrow} \mathcal{E}_{y} \rightarrow 0\right) \in \mathcal{H}$, where $\mathcal{O}_{X_{k}}(-N)=\pi^{*} \mathcal{O}_{X_{0}}(-N)$, the quotient defines an embedding $X_{k} \subset X_{0} \times G r(P(N), r)$ such that $\mathcal{E}_{y}(N)$ is the pull-back of the tautological quotient bundle. For any $g \in S L(P(N))$,

$$
g \cdot y=\left(\mathbb{C}^{P(N)} \otimes \mathcal{O}_{X_{k}}(-N) \stackrel{g}{\rightarrow} \mathbb{C}^{P(N)} \otimes \mathcal{O}_{X_{k}}(-N) \stackrel{q_{y}}{\longrightarrow} \mathcal{E}_{y} \rightarrow 0\right) \in \mathcal{H}
$$

It is easy to see that $y_{1}=g \cdot y_{2}$ if and only if $\left(\mathcal{C}_{y_{1}}, \mathcal{E}_{y_{1}}\right)$ is equivalent to $\left(\mathcal{C}_{y_{2}}, \mathcal{E}_{y_{2}}\right)$.

By $\mathbb{C}^{P(N)} \otimes \pi^{*} \mathcal{O}_{X_{0}}(-N) \stackrel{q}{\rightarrow} \mathcal{E} \rightarrow 0$, we get $\mathbb{C}^{P(N)} \otimes \mathcal{O}_{X_{0} \times \mathcal{H}}(-N) \rightarrow \pi_{*} \mathcal{E} \rightarrow 0$ on $X_{0} \times \mathcal{H}$, which induces a morphism $\theta: \mathcal{H} \rightarrow \mathcal{R}$, where $\mathcal{R}$ is the open set of $Q_{1}=\operatorname{Quot}_{P}\left(\mathcal{O}_{X_{0}}(-N)^{P(N)}\right)$ consisting of quotients $\mathcal{O}_{X_{0}}(-N)^{P(N)} \rightarrow F \rightarrow 0$ which induces $\mathbb{C}^{P(N)} \cong H^{0}(F(N))$ and $H^{1}(F(N))=0$.

Proposition 1.2 ([NS]). The morphism $\theta: \mathcal{H} \rightarrow \mathcal{R}$ is a proper $S L(P(N))$ equivariant birational morphism.

Let $\overline{\mathcal{H}}, \overline{\mathcal{R}}$ be the closure of $\mathcal{H}, \mathcal{R}$ in the Hilbert scheme and $Q_{1}$ respectively, let $\mathcal{O}_{\overline{\mathcal{H}}}(1), \mathcal{O}_{\overline{\mathcal{R}}}(1)$ be the ample line bundles pulling back from the Hilbert scheme and $Q_{1}$. Let $Z \subset \overline{\mathcal{H}} \times \overline{\mathcal{R}}$ be the closure of the graph of $\theta: \mathcal{H} \rightarrow \mathcal{R}$, and $\mathcal{O}_{Z}(a)$ be the pull-back of $\mathcal{O}_{\overline{\mathcal{H}}}(1) \otimes \mathcal{O}_{\overline{\mathcal{R}}}(a)$. Let $Z_{a}^{s}$ (resp. $Z_{a}^{s s}$ ) be the set of GIT stable (resp. semistable) points under $\mathcal{O}_{Z}(a)$. Similarly, let $\overline{\mathcal{R}}_{a}^{s}$ (resp. $\overline{\mathcal{R}}_{a}^{s s}$ ) be the set of GIT stable (resp. semistable) points under $\mathcal{O}_{\overline{\mathcal{R}}}(a)$. It is now well-known that $\overline{\mathcal{R}}_{a}^{s}=\overline{\mathcal{R}}_{a}^{s s} \subset \mathcal{R}$ is the set $\mathcal{R}^{s}:=\left\{\mathcal{O}_{X_{0}}(-N)^{P(N)} \rightarrow F \rightarrow 0 \mid F\right.$ is stable $\}$. It is 
standard that $\bar{\theta}^{-1}\left(\overline{\mathcal{R}}_{a}^{s}\right) \subset Z_{a}^{s} \subset Z_{a}^{s s} \subset \bar{\theta}^{-1}\left(\overline{\mathcal{R}}_{a}^{s s}\right)$ when $a$ is large enough, where $\bar{\theta}: Z \rightarrow \overline{\mathcal{R}}$ induced by the projection $\overline{\mathcal{H}} \times \overline{\mathcal{R}} \rightarrow \overline{\mathcal{R}}$ which coincides with $\theta$ on $\mathcal{H}$. By Proposition 1.2, $\theta$ is proper, we have $\bar{\theta}^{-1}\left(\overline{\mathcal{R}}_{a}^{s}\right)=\bar{\theta}^{-1}\left(\overline{\mathcal{R}}_{a}^{s s}\right)=\theta^{-1}\left(\mathcal{R}^{s}\right)$. Thus $Z_{a}^{s s}=Z_{a}^{s}=\theta^{-1}\left(\mathcal{R}^{s}\right)$. The projective variety structure on $G(r, d)$ of [NS] is $\mathcal{G}_{X_{0}}(r, d)=Z_{a}^{s} / / S L(P(N))$. We will show that $\mathcal{G}_{X_{0}}(r, d)$ is also the coarse moduli space of the functor defined in $[\mathrm{KL}]$ where the coarse moduli space exists as a separated and proper algebraic space.

Definition 1.3. A pair $\left(X_{k}, E\right)$ is called semistable if $\operatorname{deg}\left(\left.E\right|_{R_{i}}\right)>0$ for all $i$ and for any subsheaf $F \subset E$ we have

$$
\chi(F) \leq \frac{\chi(E)}{r} \operatorname{rank}\left(\left.F\right|_{X_{0} \backslash\left\{x_{0}\right\}}\right) .
$$

$\left(X_{k}, E\right)$ is called stable if it is semistable and when $\operatorname{rank}\left(\left.F\right|_{X_{0} \backslash\left\{x_{0}\right\}}\right) \neq 0$

$$
\chi(F)<\frac{\chi(E)}{r} \operatorname{rank}\left(\left.F\right|_{X_{0} \backslash\left\{x_{0}\right\}}\right) .
$$

Remark 1.4. In $[K L],\left(X_{k}, E\right)$ is called semistable (resp. stable) if $\operatorname{deg}\left(\left.E\right|_{R_{i}}\right)>0$ for all $i$ and for any subsheaf $F \subset E$ we have $\chi(F) \leq \frac{\chi(E)}{r} r_{\varepsilon}(F)$ (resp. $<$ ), where $\varepsilon>0$ is a sufficiently small rational number and $r_{\varepsilon}(F):=(1-\varepsilon k) \operatorname{rank}\left(\left.F\right|_{X_{0} \backslash\left\{x_{0}\right\}}\right)+$ $\varepsilon \sum_{i=1}^{k} \operatorname{rank}\left(\left.F\right|_{R_{i}}\right)$. This $\varepsilon$-stability makes sense only when $\chi(E)>0$ : For any line bundle $\left.L \subset E\right|_{R_{i}}$, the $\varepsilon$-stability implies $\operatorname{deg}(L)-1<\frac{\chi(E)}{r} \varepsilon \leq 0$ when $\chi(E) \leq 0$, which contradicts to $\operatorname{deg}\left(\left.E\right|_{R_{i}}\right)>0$. If we assume $(r, \chi(E))=1$ and $\chi(E)>0$, then the two stability are equivalent.

Lemma 1.5. If $\left(X_{k}, E\right)$ is stable, then $\left.E\right|_{R}$ is strictly standard, i.e., on each component $R_{i}$ of $R$, we have $\left.E\right|_{R_{i}}=\mathcal{O}_{R_{i}}^{a_{i}} \oplus \mathcal{O}_{R_{i}}(1)^{r-a_{i}}$ and $a_{i}<r$. Moreover, $H^{0}\left(\left.E\right|_{R}\left(-x_{1}-x_{2}\right)\right)=0$.

Proof. Let $p_{1}, p_{2}$ be the intersection points of $R_{i}$ with other components of $R$. Use stability to any line bundle $\left.L \subset E\right|_{R_{i}}$, we have $\chi\left(L\left(-p_{1}-p_{2}\right)\right)=\operatorname{deg}(L)-1 \leq 0$. On the other hand, let $F$ be the kernel of surjection $\left.E \rightarrow E\right|_{R_{i}} \rightarrow L$. Then, by stability of $E, \chi(L)=\chi(E)-\chi(F)>0$. Thus $0 \leq \operatorname{deg}(L) \leq 1$.

If there is $s \in H^{0}\left(\left.E\right|_{R}\left(-x_{1}-x_{2}\right)\right)$ with $s \neq 0$, then $s$ generates a subsheaf $\left.\mathcal{L} \subset E\right|_{R}\left(-x_{1}-x_{2}\right) \subset E$ with $\chi(\mathcal{L}) \geq 1$. By stability of $E, \chi(\mathcal{L}) \leq 0$. This is a contradiction.

Proposition 1.6. Let $\pi: X_{k} \rightarrow X_{0}$ be the canonical morphism contracting the chain $R$ of projective lines. If $\left(X_{k}, E\right)$ is semistable (resp. stable), then $\pi_{*}(E)$ is semistable (resp. stable). If $\left.E\right|_{R}$ is strictly positive and $\pi_{*}(E)$ is semistable (resp. stable). then $\left(X_{k}, E\right)$ is semistable (resp. stable).

Proof. Assume $k>0$, let $X_{k}=\widetilde{X}_{0} \cup R, \widetilde{X}_{0} \cap R=\left\{x_{1}, x_{2}\right\}, \widetilde{E}=\left.E\right|_{\widetilde{X}_{0}}, \widetilde{F}=\left.E\right|_{R}$. Consider $E$ being obtained by isomorphisms $\widetilde{F}_{x_{1}^{\prime}} \stackrel{\theta_{1}}{\longrightarrow} \widetilde{E}_{x_{1}}, \widetilde{F}_{x_{2}^{\prime}} \stackrel{\theta_{2}}{\longrightarrow} \widetilde{E}_{x_{2}}$. Then

$$
H^{0}(R, \widetilde{F}) \stackrel{s \mapsto\left(s\left(x_{1}^{\prime}\right), s\left(x_{2}^{\prime}\right)\right)}{\longrightarrow} \widetilde{F}_{x_{1}^{\prime}} \oplus \widetilde{F}_{x_{2}^{\prime}} \stackrel{\theta_{1} \oplus \theta_{2}}{\longrightarrow} \widetilde{E}_{x_{1}} \oplus \widetilde{E}_{x_{2}}
$$

gives the GPB on $\widetilde{X}_{0}$, which defines $\pi_{*}(E)$ as follows: the canonical exact sequence $0 \rightarrow \widetilde{F}\left(-x_{1}-x_{2}\right) \rightarrow E \rightarrow \widetilde{E} \rightarrow 0$ induces the defining sequence

$$
0 \rightarrow \pi_{*}(E) \rightarrow \pi_{*}(\widetilde{E}) \rightarrow x_{0} H^{1}\left(\widetilde{F}\left(-x_{1}-x_{2}\right)\right) \rightarrow 0 .
$$


It is enough to show that this GPB is semistable (resp. stable) when $\left(X_{k}, E\right)$ semistable (resp. stable). For any subsheaf $\widetilde{E}^{\prime} \subset \widetilde{E}\left(\right.$ on $\left.\widetilde{X}_{0}\right)$, let $j_{i}: \widetilde{E}_{x_{i}}^{\prime} \rightarrow \widetilde{E}_{x_{i}}$ and

$$
K=\left\{s \in H^{0}(R, \widetilde{F}) \mid\left(\theta_{1}\left(s\left(x_{1}^{\prime}\right)\right), \theta_{2}\left(s\left(x_{2}^{\prime}\right)\right)\right) \in j_{1}\left(\widetilde{E}_{x_{1}}\right) \oplus j_{2}\left(\widetilde{E}_{x_{2}}\right)\right\} .
$$

Then, if $r_{1}=\operatorname{dim}\left(j_{1}\left(\widetilde{E}_{x_{1}}^{\prime}\right)\right), r_{2}=\operatorname{dim}\left(j_{2}\left(\widetilde{E}_{x_{2}}^{\prime}\right)\right)$, it is enough to show

$$
\frac{\chi\left(\widetilde{E}^{\prime}\right)+\operatorname{dim}(K)-r_{1}-r_{2}}{\operatorname{rank}\left(\widetilde{E}^{\prime}\right)} \leq \frac{\chi(\widetilde{E})+\operatorname{dim}\left(H^{0}(R, \widetilde{F})\right)-2 r}{r} \quad(\text { resp. }<) .
$$

Let $\mathcal{K} \subset \widetilde{F}$ be the subsheaf generated by $K \subset H^{0}(R, \widetilde{F})$ (thus $H^{1}(\mathcal{K})=0$ ), we are going to glue $\widetilde{E}^{\prime}$ and $\mathcal{K}$ into a subsheaf $E^{\prime} \subset E$. By the choice of $K$, the diagram

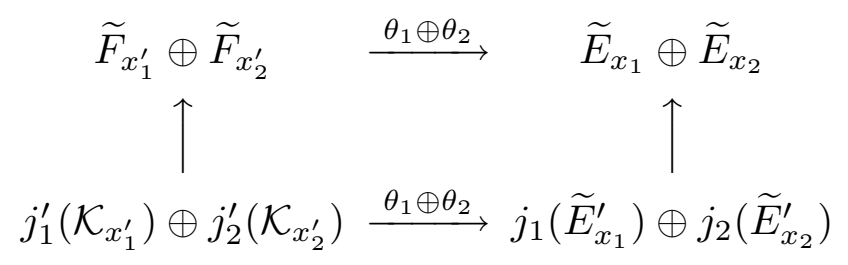

is commutative, where $j_{i}^{\prime}: \mathcal{K}_{x_{i}^{\prime}} \rightarrow \widetilde{F}_{x_{i}^{\prime}}$ is the map induced by $\mathcal{K} \subset \widetilde{F}$. Let $\varphi$ : $\widetilde{X}_{0} \sqcup R \rightarrow X_{k}$ be the gluing morphism. Then there is a subsheaf $E^{\prime} \subset E$ defined by

$$
0 \rightarrow E^{\prime} \rightarrow \varphi_{*}\left(\mathcal{K} \oplus \widetilde{E}^{\prime}\right) \rightarrow{ }_{x_{1}} j_{1}\left(\widetilde{E}_{x_{1}}^{\prime}\right) \oplus{ }_{x_{2}} j_{2}\left(\widetilde{E}_{x_{2}}^{\prime}\right) \rightarrow 0 .
$$

Since $\chi\left(E^{\prime}\right)=\chi\left(\widetilde{E}^{\prime}\right)+\chi(\mathcal{K})-r_{1}-r_{2}=\chi\left(\widetilde{E}^{\prime}\right)+\operatorname{dim}\left(H^{0}(R, \mathcal{K})\right)-r_{1}-r_{2}$ and $\chi(E)=\chi(\widetilde{E})+\operatorname{dim}\left(H^{0}(R, \widetilde{F})\right)-2 r$, semistability (resp. stability) of $\left(X_{k} . E\right)$ implies

$$
\frac{\chi\left(\widetilde{E}^{\prime}\right)+\operatorname{dim}\left(H^{0}(R, \mathcal{K})\right)-r_{1}-r_{2}}{\operatorname{rank}\left(\widetilde{E}^{\prime}\right)} \leq \frac{\chi(\widetilde{E})+\operatorname{dim}\left(H^{0}(R, \widetilde{F})\right)-2 r}{r} \quad(\text { resp. }<) .
$$

Thus $\pi_{*}(E)$ is semistable (resp. stable).

Assume $\pi_{*}(E)$ semistable (resp. stable), for any $E_{1} \subset E$, we need to show

$$
\chi\left(E_{1}\right) \leq \frac{\chi(E)}{r} \operatorname{rank}\left(\left.E_{1}\right|_{\tilde{X}_{0}}\right)
$$

$\left(\operatorname{resp} . \chi\left(E_{1}\right)<\frac{\chi(E)}{r} \operatorname{rank}\left(\left.E_{1}\right|_{\tilde{X}_{0}}\right)\right.$ when $\left.\operatorname{rank}\left(\left.E_{1}\right|_{\tilde{X}_{0}}\right) \neq 0\right)$. Consider exact sequence

$$
0 \rightarrow \widetilde{F}\left(-x_{1}-x_{2}\right) \rightarrow E \rightarrow \widetilde{E} \rightarrow 0
$$

let $\widetilde{E}_{1} \subset \widetilde{E}$ be the image of $E_{1}$ and $\mathcal{K} \subset \widetilde{F}\left(-x_{1}-x_{2}\right)$ be the kernel of $E_{1} \rightarrow \widetilde{E}_{1}$. Then we have $0 \rightarrow \pi_{*}\left(E_{1}\right) \rightarrow \pi_{*}\left(\widetilde{E}_{1}\right) \rightarrow R^{1} \pi_{*}(\mathcal{K})={ }_{x_{0}} H^{1}(\mathcal{K})$ since $\pi_{*} \widetilde{F}\left(-x_{1}-\right.$ $\left.x_{2}\right)=0$. Thus $\chi\left(E_{1}\right)=\chi\left(\widetilde{E}_{1}\right)+\chi(\mathcal{K})=\chi\left(\pi_{*} \widetilde{E}_{1}\right)-\operatorname{dim}\left(H^{1}(\mathcal{K})\right) \leq \chi\left(\pi_{*} E_{1}\right)$. The assumption of $\left.E\right|_{R}$ being positive implies $\chi\left(\pi_{*} E\right)=\chi(E)$. Then the semistability (resp. stability) of $\pi_{*}(E)$ implies the semistability (resp. stability) of ( $\left.X_{k}, E\right)$.

For any scheme $T$, a family $\left(\mathcal{X}_{T}, \mathcal{E}_{T}\right)$ of stable Gieseker bundles of rank $r$ and degree $d$ over $T$ consists (1) a flat family $f_{T}: \mathcal{X}_{T} \rightarrow T$ of curves $X_{k}(0 \leq k \leq r)$ with the canonical morphism $\pi: \mathcal{X}_{T} \rightarrow X_{0} \times T$ contracting the chains of projective 
lines such that $f_{T}=p_{T} \cdot \pi$ where $p_{T}: X_{0} \times T \rightarrow T$ is the projection, (2) a rank $r$ vector bundle $\mathcal{E}_{T}$ on $\mathcal{X}_{T}$ such that on each fiber $\mathcal{E}_{T}$ is stable of Euler characteristic $\chi=d+r(1-g)$. Two families $\left(\mathcal{X}_{T}, \mathcal{E}_{T}\right)$ and $\left(\mathcal{X}_{T}^{\prime}, \mathcal{E}_{T}^{\prime}\right)$ are equivalent if there is an $T$-isomorphism $\sigma_{T}: \mathcal{X}_{T} \rightarrow \mathcal{X}_{T}^{\prime}$ and a line bundle $\mathcal{L}_{T}$ on $T$ such that

$$
\pi=\pi^{\prime} \cdot \sigma_{T}, \quad \sigma_{T}^{*}\left(\mathcal{E}_{T}^{\prime}\right) \cong \mathcal{E}_{T} \otimes f_{T}^{*}\left(\mathcal{L}_{T}\right)
$$

Define the moduli functor

$$
\underline{\mathrm{GV}^{s}}(r, \chi):\{\text { Schemes }\} \rightarrow\{\text { Sets }\}
$$

for any scheme $T, \mathrm{GV}^{s}(r, \chi)(T)$ is the set of equivalence classes of families $\left(\mathcal{X}_{T}, \mathcal{E}_{T}\right)$ of stable Gieseker bundles of rank $r$ and Euler characteristic $\chi=d+r(1-g)$.

Theorem 1.7. When $(r, \chi)=1$, the projective variety $\mathcal{G}_{X_{0}}(r, d)$ corepresents the functor $G V^{s}(r, \chi)$. That is, there is a natural transformation

$$
\Phi: \underline{G V^{s}}(r, \chi) \rightarrow H o m\left(\bullet, \mathcal{G}_{X_{0}}(r, d)\right)
$$

of functors such that for any other scheme $M$ and any other natural transformation

$$
\Phi^{\prime}: \underline{G V^{s}}(r, \chi) \rightarrow H o m(\bullet, M)
$$

there is a unique morphism $t: \mathcal{G}_{X_{0}}(r, d) \rightarrow M$ satisfying $\Phi^{\prime}=h(t) \cdot \Phi$.

Proof. To define $\Phi$, we define for any scheme $T$ the map

$$
\Phi_{T}: \underline{\mathrm{GV}}^{s}(r, \chi)(T) \rightarrow \operatorname{Hom}\left(T, \mathcal{G}_{X_{0}}(r, d)\right)
$$

For any $\left(\mathcal{X}_{T}, \mathcal{E}_{T}\right) \in \underline{G V}^{s}(r, \chi)(T)$, let $\left\{T_{i}\right\}_{i \in I}$ be an affine covering of $T$ such that $f_{T *} \mathcal{E}_{T}(N)$ is trivial on each $T_{i}$. If we fix a trivialization on each $T_{i}$, we get morphisms $\phi_{\left(\mathcal{X}_{T_{i}}, \mathcal{E}_{T_{i}}\right)}: T_{i} \rightarrow Z_{a}^{s}$ such that $\mathcal{E}_{T_{i}}$ is the pullback of the universal quotient $C^{P(N)} \otimes$ $\pi^{*} \mathcal{O}_{X_{0}}(-N) \stackrel{q}{\rightarrow} \mathcal{E} \rightarrow 0$ on $\mathcal{C}$. It is clear that these morphisms define a morphism

$$
\phi_{\left(\mathcal{X}_{T}, \mathcal{E}_{T}\right)}: T \rightarrow Z_{a}^{s} / / S L(P(N))=\mathcal{G}_{X_{0}}(r, d)
$$

since the dependences of trivializations are precisely modulated by the group action. Define $\Phi_{T}\left(\left(\mathcal{X}_{T}, \mathcal{E}_{T}\right)\right)=\phi_{\left(\mathcal{X}_{T}, \mathcal{E}_{T}\right)} \in \operatorname{Hom}\left(T, \mathcal{G}_{X_{0}}(r, d)\right)$, we get the natural transformation

$$
\Phi: \underline{\mathrm{GV}}^{s}(r, \chi) \rightarrow \operatorname{Hom}\left(\bullet, \mathcal{G}_{X_{0}}(r, d)\right) .
$$

For any other scheme $M$ and any other natural transformation

$$
\Phi^{\prime}: \underline{\mathrm{GV}^{s}}(r, \chi) \rightarrow \operatorname{Hom}(\bullet, M),
$$

the unique morphism $t: \mathcal{G}_{X_{0}}(r, d) \rightarrow M$ is induced by the morphism $\Phi_{Z_{a}^{s}}^{\prime}((\mathcal{C}, \mathcal{E})) \in$ $\operatorname{Hom}\left(Z_{a}^{s}, M\right)$, where $(\mathcal{C}, \mathcal{E}) \in \underline{\mathrm{GV}}^{s}(r, \chi)\left(Z_{a}^{s}\right)$ is the universal object. 


\section{$\S 2$ The normalization of Generalized Gieseker moduli spaces}

Recall that $\mathcal{H} \subset \operatorname{Hilb}^{P_{1}}\left(X_{0} \times G r(P(N), r)\right)$ is the $S L(P(N))$-stable subscheme parametrizes the curves in $\operatorname{Gr}(P(N), r)$ of type $X_{k}$, and $\mathcal{C} \subset X_{0} \times Y \times G r(P(N), r)$ is the universal curve. The action of $S L(P(N))$ on $X_{0} \times \mathcal{H} \times G r(P(N), r)$ induces clearly an action on $\mathcal{C}$ such that the projection $p_{2}: \mathcal{C} \rightarrow \mathcal{H}$ is a $S L(P(N))$-linear morphism. It is known that the subscheme $\widetilde{\mathcal{H}} \subset \mathcal{C}$ defined by the first Fitting ideal of the sheaf $\Omega_{\mathcal{C} / \mathcal{H}}^{1}$ is the normalization of $\mathcal{H}$ (See [K2], proof of Theorem 4.9).

Lemma 2.1. The smooth variety $\widetilde{\mathcal{H}}$ represents a functor

$$
\widetilde{\mathcal{Y}}:(\mathbb{C} \text {-schemes }) \rightarrow(\text { Sets }),
$$

where $\tilde{\mathcal{Y}}(T)$ is defined to be the set of pairs $\left(\mathcal{C}_{T}, s_{T}\right), \mathcal{C}_{T} \in \mathcal{Y}(T)$ and $s_{T}: T \rightarrow \mathcal{C}_{T}$ is a section of nodes in the fibres of $\mathcal{C}_{T} \rightarrow T$.

Proof. Straightforward.

The normalization $p_{2}: \widetilde{\mathcal{H}} \rightarrow \mathcal{H}$ is a $S L(P(N))$-linear morphism. Recall in Section 1 we have compactified $\mathcal{H}$ by a projective variety $Z$ and have chosen an ample line bundle $\mathcal{O}_{Z}(a)$ so that $Z_{a}^{s s}=Z_{a}^{s} \subset \mathcal{H}$ and $\mathcal{G}_{X_{0}}(r, d)=Z_{a}^{s} / / S L(P(N))$. We can similarly compactify $\widetilde{\mathcal{H}}$ by a projective variety $\widetilde{Z}$ and choose an ample line bundle $\mathcal{O}_{\widetilde{Z}}(a)$ so that $\widetilde{Z}_{a}^{s s}=\widetilde{Z}_{a}^{s}=p_{2}^{-1}\left(Z_{a}^{s}\right) \subset \widetilde{\mathcal{H}}$. Thus $\mathcal{N}_{\widetilde{X}_{0}}(r, d)=\widetilde{Z}_{a}^{s} / / S L(P(N))$ is the normalization of $\mathcal{G}_{X_{0}}(r, d)$. The smooth projective variety $\mathcal{N}_{\widetilde{X}_{0}}(r, d)$ itself is a moduli space of marked stable Gieseker bundles that we are going to define.

For any scheme $T$, a family $\left(\mathcal{X}_{T}, \mathcal{E}_{T}, s_{T}\right)$ of marked stable Gieseker bundles of rank $r$ and degree $d$ over $T$ consists (1) a flat family $f_{T}: \mathcal{X}_{T} \rightarrow T$ of curves $X_{k}$ $(0 \leq k \leq r)$ with the canonical morphism $\pi: \mathcal{X}_{T} \rightarrow X_{0} \times T$ contracting the chains of projective lines such that $f_{T}=p_{T} \cdot \pi$ where $p_{T}: X_{0} \times T \rightarrow T$ is the projection, (2) a rank $r$ vector bundle $\mathcal{E}_{T}$ on $\mathcal{X}_{T}$ such that on each fiber $\mathcal{E}_{T}$ is stable of Euler characteristic $\chi=d+r(1-g)$, (3) a section $s_{T}: T \rightarrow \mathcal{X}_{T}$ of nodes in the fibres of $f_{T}: \mathcal{X}_{T} \rightarrow T$. Two families $\left(\mathcal{X}_{T}, \mathcal{E}_{T}, s_{T}\right)$ and $\left(\mathcal{X}_{T}^{\prime}, \mathcal{E}_{T}^{\prime}, s_{T}^{\prime}\right)$ are equivalent if there is an $T$-isomorphism $\sigma_{T}: \mathcal{X}_{T} \rightarrow \mathcal{X}_{T}^{\prime}$ and a line bundle $\mathcal{L}_{T}$ on $T$ such that

$$
\pi=\pi^{\prime} \cdot \sigma_{T}, \quad \sigma_{T} \cdot s_{T}=s_{T}^{\prime}, \quad \sigma_{T}^{*}\left(\mathcal{E}_{T}^{\prime}\right) \cong \mathcal{E}_{T} \otimes f_{T}^{*}\left(\mathcal{L}_{T}\right) .
$$

Define the moduli functor

$$
\underline{\mathrm{GV}}^{m s}(r, \chi):\{\text { Schemes }\} \rightarrow\{\text { Sets }\}
$$

for any scheme $T, \underline{G V}^{m s}(r, \chi)(T)$ is the set of equivalence classes of families of marked stable Gieseker bundles of rank $r$ and Euler characteristic $\chi=d+r(1-g)$.

Theorem 2.2. When $(r, \chi)=1$, the projective variety $\mathcal{N}_{\widetilde{X}_{0}}(r, d)$ corepresents the functor $G V^{m s}(r, \chi)$. That is, there is a natural transformation

$$
\Phi: \underline{G V}^{m s}(r, \chi) \rightarrow \operatorname{Hom}\left(\bullet, \mathcal{N}_{\widetilde{X}_{0}}(r, d)\right)
$$

of functors such that for any other scheme $M$ and any other natural transformation

$$
\Phi^{\prime}: \underline{G V}^{m s}(r, \chi) \rightarrow H o m(\bullet, M)
$$


there is a unique morphism $t: \mathcal{N}_{\widetilde{X}_{0}}(r, d) \rightarrow M$ satisfying $\Phi^{\prime}=h(t) \cdot \Phi$.

Proof. Straightforward and similarly with the proof of Theorem 1.7.

In the rest of this section, we will give another construction of $\mathcal{N}_{\widetilde{X}_{0}}(r, d)$. In fact, we will construct a sequence $\left\{M^{\alpha}\right\}_{0<\alpha<1}$ of GIT quotients such that $M^{1^{-}}$is $\mathcal{N}_{\widetilde{X}_{0}}(r, d)$ and $M^{0^{+}}$is a fibre bundle over $\mathcal{U}_{\widetilde{X}_{0}}(r, d)$ with fibre being the wonderful compactification of $G L(r)$, where $\ell^{-}$(resp. $\ell^{+}$) denote rational numbers which are smaller (resp. bigger) than $\ell$ and are sufficiently closing to $\ell$. We give firstly an interpretation of the functor $\mathrm{GV}^{m s}(r, \chi)$ from another point of view.

Recall $\widetilde{X}_{0}$ being the normalization of $X_{0}$ and $x_{1}, x_{2}$ being the two preimages of the node. Follow the notation of [K2], a modification of $\left(\widetilde{X}_{0}, x_{1}, x_{2}\right)$ at $x_{1}$ and $x_{2}$ is a morphism of two pointed curves $\left(Y_{n, m}, y_{1}, y_{2}\right) \stackrel{h}{\rightarrow}\left(\tilde{X}_{0}, x_{1}, x_{2}\right)$ where

$$
Y_{n, m}=\widetilde{X}_{0} \cup R^{\prime} \cup R^{\prime \prime}, \quad R^{\prime}=\bigcup_{i=1}^{n} R_{i}^{\prime}, \quad R^{\prime \prime}=\bigcup_{i=1}^{m} R_{i}^{\prime \prime}
$$

$R^{\prime}, R^{\prime \prime}$ are chains of $\mathbb{P}^{1}$ s of length $n, m$ such that $h\left(R^{\prime}\right)=x_{1}, h\left(R^{\prime \prime}\right)=x_{2}, y_{1} \in R_{n}^{\prime}$, $y_{2} \in R_{m}^{\prime \prime}$ are smooth points of $Y_{n, m}$ and $R^{\prime} \cap \widetilde{X}_{0}=R_{1}^{\prime} \cap \widetilde{X}_{0}=\left\{x_{1}\right\}, R^{\prime \prime} \cap \widetilde{X}_{0}=$ $R_{1}^{\prime \prime} \cap \widetilde{X}_{0}=\left\{x_{2}\right\}$. A GPB of rank $r$ and degree $d$ on $Y_{n, m}$ consists of a vector bundle $V$ of rank $r$ and degree $d$ on $Y_{n, m}$ with an isomorphism $\varphi: V_{y_{1}} \cong V_{y_{2}}$. Let $\Gamma_{\varphi} \subset V_{y_{1}} \oplus V_{y_{2}}$ be the graph of $\varphi$. Then we can define a marked Gieseker bundle $\left(X_{n+m}, y, E\right)$ of rank $r$ and Euler characteristic $\chi=d+r(1-g)$ as follows

(1) Identify $y_{1}, y_{2}$ into a point $y$, we get $j:\left(Y_{n, m}, y_{1}, y_{2}\right) \rightarrow\left(X_{n+m}, y\right)$.

(2) The bundle $E$ on $X_{n+m}$ is defined by exact sequence

$$
0 \rightarrow E \rightarrow j_{*}(V) \rightarrow y \frac{V_{y_{1}} \oplus V_{y_{2}}}{\Gamma_{\varphi}} \rightarrow 0 .
$$

Follow [K2], $\left(Y_{n, m}, y_{1}, y_{2}, V, \Gamma_{\varphi}\right)$ is called a Gieseker vector bundle data.

Definition 2.3. For any subsheaf $V^{\prime} \subset V$, let $r_{0}\left(V^{\prime}\right)$ denote the rank of $\left.V^{\prime}\right|_{\widetilde{X}_{0}}$. When $r_{0}\left(V^{\prime}\right) \neq 0$ and $V / V^{\prime}$ torsion free, let

$$
\mu_{\alpha}\left(V^{\prime}\right):=\frac{\chi\left(V^{\prime}\right)-(1-\alpha) \operatorname{dim}\left(V_{y_{1}}^{\prime} \oplus V_{y_{2}}^{\prime}\right)-\alpha \operatorname{dim} \frac{V_{y_{1}}^{\prime} \oplus V_{y_{2}}^{\prime}}{\left(V_{y_{1}}^{\prime} \oplus V_{y_{2}}^{\prime}\right) \cap \Gamma_{\varphi}}}{r_{0}\left(V^{\prime}\right)} .
$$

Then the Gieseker vector bundle data $\left(Y_{n, m}, y_{1}, y_{2}, V, \Gamma_{\varphi}\right)$ is called $\alpha$-semistable (resp. $\alpha$-stable) if $V$ has positive degree on each projective line,

$$
\mu_{\alpha}\left(V^{\prime}\right) \leq \mu_{\alpha}(V)=\frac{\chi(V)-(2-\alpha) r}{r} \quad(\text { resp. }<),
$$

and $\chi\left(V^{\prime}\right)-\operatorname{dim} \frac{V_{y_{1}}^{\prime} \oplus V_{y_{2}}^{\prime}}{\left(V_{y_{1}}^{\prime} \oplus V_{y_{2}}^{\prime}\right) \cap \Gamma_{\varphi}} \leq 0$ when $r_{0}\left(V^{\prime}\right)=0$.

Remark 2.4. For $\alpha \in[0,1]$, there may exist $V^{\prime} \subset V$ with $r_{0}\left(V^{\prime}\right) \neq 0$ such that the equality in Definition 2.3 holds. These critical points form a finite subset $I_{r} \subset[0,1]$. For any $\alpha \in[0,1] \backslash I_{r}, \alpha$-semistability is equivalent to $\alpha$-stability. It is clear that 0 and 1 are not in $I_{r}$ when $(r, d)=1$. 
Lemma 2.5. If $(r, d)=1$, then $\left(Y_{n, m}, y_{1}, y_{2}, V, \Gamma_{\varphi}\right)$ is $1^{-}$-stable if and only if the associated marked Gieseker bundle $\left(X_{n+m}, y, E\right)$ is stable.

Proof. A straightforward computation.

We can define familes of $\alpha$-stable Gieseker vector bundle data over a scheme $T$ (See Definition 4.7 of [K2] for details). It consists of (1) a modification $\left(\mathcal{Y}, s_{1}, s_{2}, h\right)$ of $\left(\widetilde{X}_{0}, x_{1}, x_{2}\right)$ over $T$ that is a commutative diagram

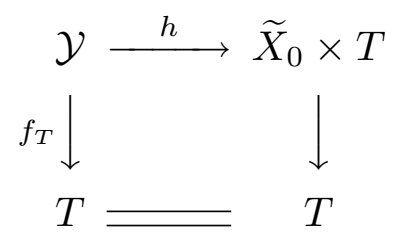

with two sections $s_{i}: T \rightarrow \mathcal{Y}(i=1,2)$ such that $\left(\mathcal{Y}_{t}, s_{1}(t), s_{2}(t), h_{t}\right)$ is a modification of $\left(\widetilde{X}_{0}, x_{1}, x_{2}\right)$ for any $t \in T,(2)$ a vector bundle $\mathcal{V}$ of rank $r$ and degree $d$ on $\mathcal{Y}$ and an isomorphism $\varphi: s_{1}^{*} \mathcal{V} \cong s_{2}^{*} \mathcal{V}$ such that $\left(\mathcal{Y}_{t}, s_{1}(t), s_{2}(t), \mathcal{V}_{t}, \varphi_{t}\right)$ is $\alpha$-stable for any $t \in T$. Two families $\left(\mathcal{Y}, s_{1}, s_{2}, h, \mathcal{V}, \varphi\right)$ and $\left(\mathcal{Y}^{\prime}, s_{1}^{\prime}, s_{2}^{\prime}, h^{\prime}, \mathcal{V}^{\prime}, \varphi^{\prime}\right)$ are equivalent if there is an $T$-isomorphism $\sigma_{T}: \mathcal{Y} \rightarrow \mathcal{Y}^{\prime}$ and a line bundle $\mathcal{L}_{T}$ on $T$ such that $h=h^{\prime} \cdot \sigma_{T}, \sigma_{T} \cdot s_{i}=s_{i}^{\prime}(i=1,2)$ and $\sigma_{T}^{*}\left(\mathcal{V}^{\prime}\right) \cong \mathcal{V} \otimes f_{T}^{*}\left(\mathcal{L}_{T}\right)$, where the isomorphisms satisfy the commutative diagram

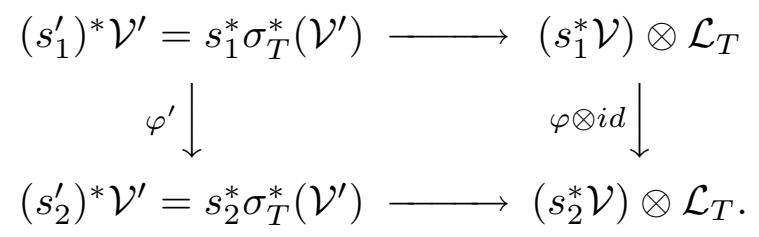

Define the functor

$$
\underline{\mathrm{GVD}}^{\alpha}(r, \tilde{\chi}):\{\text { Schemes }\} \rightarrow\{\text { Sets }\}
$$

$\mathrm{GVD}^{\alpha}(r, \tilde{\chi})(T)$ is the set of equivalence classes of families of $\alpha$-stable Gieseker vector bundle data of rank $r$ and Euler characteristic $\tilde{\chi}=\chi+r$.

Lemma 2.6. When $\alpha=1^{-}$, the functor $G V D^{\alpha}(r, \widetilde{\chi})$ is canonically isomorphic to the functor $G V^{m s}(r, \chi)$ of marked stable Gieseker bundles.

Proof. For any $\left(\mathcal{Y}, s_{1}, s_{2}, h, \mathcal{V}, \varphi\right) \in \underline{\operatorname{GVD}}^{\alpha}(r, \widetilde{\chi})(T)$, by identifying the two sections $s_{1}$ and $s_{2}$ (cf. [K2]), we get $T$-morphism $j:\left(\mathcal{Y}, s_{1}, s_{2}\right) \rightarrow(\mathcal{X}, s)$ and a vector bundle $\mathcal{E}$ on $\mathcal{X}$ defined by

$$
0 \rightarrow \mathcal{E} \rightarrow j_{*}(\mathcal{V}) \rightarrow s(T) \frac{s_{1}^{*} \mathcal{V} \oplus s_{2}^{*} \mathcal{V}}{\Gamma_{\varphi}} \rightarrow 0
$$

By Lemma $2.5,(\mathcal{X}, \mathcal{E}, s)$ is a family of marked stable Gieseker bundles. This defines the canonical isomorphism

$$
\underline{\mathrm{GVD}}^{\alpha}(r, \tilde{\chi}) \rightarrow \underline{\mathrm{GV}}^{m s}(r, \chi)
$$

whose inverse is defined as follows: for any $(\mathcal{X}, \mathcal{E}, s) \in \underline{\mathrm{GV}}^{m s}(r, \chi)(T)$, let

$$
\left(\mathcal{Y}, s_{1}, s_{2}\right) \stackrel{h}{\rightarrow} \widetilde{X}_{0} \times T
$$


be the pull-back of $(\mathcal{X}, s) \rightarrow X_{0} \times T$ by the base change $\tilde{X}_{0} \times T \rightarrow X_{0} \times T, \mathcal{V}$ be the pull-back of $\mathcal{E}$ and $\varphi$ the natural identification. Then, when $\alpha=1^{-}$,

$$
\left(\mathcal{Y}, s_{1}, s_{2}, h, \mathcal{V}, \varphi\right) \in \underline{\mathrm{GVD}}^{\alpha}(r, \tilde{\chi})(T) .
$$

Let $\widetilde{P}(N)=r N+\widetilde{\chi}, \mathbf{Q} \subset \operatorname{Quot}_{\widetilde{P}}\left(\mathcal{O}_{\widetilde{X}_{0}}(-N)^{\widetilde{P}(N)}\right)$ be the open subscheme of Quot scheme consisting of locally free quotients and $\mathcal{F}$ be the universal quotient on $\widetilde{X}_{0} \times \mathbf{Q}$. Let $\mathbf{P}^{0}=\mathbb{P}\left(\left(\mathcal{H} \text { om }\left(\mathcal{F}_{x_{1}}, \mathcal{F}_{x_{2}}\right) \oplus \mathcal{O}_{\mathbf{Q}}\right)^{\vee}\right) \rightarrow \mathbf{Q}$. It contains the open subvariety $\mathbb{V}\left(\mathcal{H o m}\left(\mathcal{F}_{x_{1}}, \mathcal{F}_{x_{2}}\right)^{\vee}\right)$. For any scheme $T$, the $T$-valued points of $\mathbb{V}\left(\mathcal{H} \operatorname{om}\left(\mathcal{F}_{x_{1}}, \mathcal{F}_{x_{2}}\right)^{\vee}\right)$ are the pairs $(x, \xi)$ where $x: T \rightarrow \mathbf{Q}$ is a morphism of schemes and $\xi: x^{*} \mathcal{F}_{x_{1}} \rightarrow$ $x^{*} \mathcal{F}_{x_{2}}$ is a morphism of $\mathcal{O}_{T}$-modules. For $i \in[0, r-1]$, let $Y_{i}^{\prime} \subset \mathbb{V}\left(\mathcal{H o m}\left(\mathcal{F}_{x_{1}}, \mathcal{F}_{x_{2}}\right)^{\vee}\right)$ be the closed subvariety whose $T$-valued points are $(x, \xi)$ where $\xi$ is of rank at most $i$ and let $Y_{i}^{0}$ be the closure of $Y_{i}^{\prime}$ in $\mathbf{P}^{0}$. Let $Z_{0}^{0}$ be the complement of $\mathbb{V}\left(\mathcal{H o m}\left(\mathcal{F}_{x_{1}}, \mathcal{F}_{x_{2}}\right)^{\vee}\right)$ inside $\mathbf{P}^{0}$ and define $Z_{i}^{0}$ to be the intersection of $Y_{r-i}^{0}$ with $Z_{0}^{0}(i \in[1, r-1])$. Now $\mathbf{H}=K G L\left(\mathcal{F}_{x_{1}}, \mathcal{F}_{x_{2}}\right)$ may be defined as the result of the following successive blowing ups:

$$
\mathbf{H}=K G L\left(\mathcal{F}_{x_{1}}, \mathcal{F}_{x_{2}}\right)=\mathbf{P}^{r-1} \rightarrow \mathbf{P}^{r-2} \rightarrow \cdots \rightarrow \mathbf{P}^{1} \rightarrow \mathbf{P}^{0} .
$$

Here, $\mathbf{P}^{1}$ is the blow up of $\mathbf{P}^{0}$ along the union of $Y_{0}^{0}$ and $Z_{n-1}^{0}$ (one might equally well blow up first along $Y_{0}^{0}$ and then along $Z_{n-1}^{0}$ or vice versa since these subvarieties are disjoint). Now denote by $Y_{i}^{1}, Z_{i}^{1}$ the proper transforms of $Y_{i}^{0}, Z_{i}^{0}$. Then $Y_{1}^{1}$ and $Z_{n-2}^{1}$ are disjoint and smooth and $\mathbf{P}^{2}$ is the blow up of $\mathbf{P}^{1}$ along $Y_{1}^{1} \cup Z_{n-2}^{1}$. The general step consists in blowing up $\mathbf{P}^{i}$ along $Y_{i}^{i} \cup Z_{n-i-1}^{i}$. According to [K1], $\mathbf{H}=K G L\left(\mathcal{F}_{x_{1}}, \mathcal{F}_{x_{2}}\right)$ has a modular interpretation. The $T$-valued points of $\mathbf{H}=$ $K G L\left(\mathcal{F}_{x_{1}}, \mathcal{F}_{x_{2}}\right)$ consist of pairs $(x, \xi)$, where $x: T \rightarrow \mathbf{Q}$ is a morphism of schemes and $\xi$ is (an equivalence class of) a generalized isomorphism from $x^{*} \mathcal{F}_{x_{1}}$ to $x^{*} \mathcal{F}_{x_{2}}$. In [K2], Ivan Kausz proved further that the functor of Gieseker vector bundle data is isomorphic to the functor of generalized isomorphisms. In particular, there is firstly a modification $\left(\mathcal{C} \rightarrow \mathbf{H}, s_{1}, s_{2}\right) \stackrel{h}{\rightarrow}\left(\widetilde{X}_{0} \times \mathbf{H}, x_{1}, x_{2}\right)$ of $\left(\widetilde{X}_{0}, x_{1}, x_{2}\right)$ over $\mathbf{H}$ (see Definition 4.4 of [K2]), where $s_{i}: \mathbf{H} \rightarrow \mathcal{C}(i=1,2)$ are sections that map (under $h$ ) to the sections $\left\{x_{i}\right\} \times \mathbf{H}(i=1,2)$. Secondly, there is an admissible $(\mathcal{E}, \varphi)$ on $\mathcal{C}$ such that $h_{\bullet}(\mathcal{E}) \cong \mathcal{F}$ on $\widetilde{X}_{0} \times \mathbf{H}$.

Let $\mathbf{G}=\operatorname{Grass}_{r}\left(\mathcal{F}_{x_{1}}, \oplus \mathcal{F}_{x_{2}}\right) \rightarrow \mathbf{Q}$ be the Grassmanian variety over $\mathbf{Q}$ which parametrizes subbundles of rank $r$ of $\mathcal{F}_{x_{1}} \oplus \mathcal{F}_{x_{2}}$. By Proposition 10.1 of [K1], there is a proper birational $\mathbf{Q}$-morphism

$$
f: \mathbf{H}=K G L\left(\mathcal{F}_{x_{1}}, \mathcal{F}_{x_{2}}\right) \rightarrow \mathbf{G}=\operatorname{Grass}_{r}\left(\mathcal{F}_{x_{1}}, \oplus \mathcal{F}_{x_{2}}\right) .
$$

To see the birational morphism $f: \mathbf{H} \rightarrow \mathbf{G}$, let $p \in \mathbf{H}$ be a closed point. Then the fibre $\mathcal{C}_{p}=\widetilde{X}_{0} \cup R^{\prime} \cup R^{\prime \prime}$ is the curve $\widetilde{X}_{0}$ attached two disjoint chains $R^{\prime}, R^{\prime \prime}$ of rational lines at $x_{1}, x_{2}$ with two marked points $y_{1} \in R_{n}^{\prime}, y_{2} \in R_{m}^{\prime \prime}$, where

$$
R^{\prime}=\bigcup_{i=1}^{n} R_{i}^{\prime}, \quad R^{\prime \prime}=\bigcup_{i=1}^{m} R_{i}^{\prime \prime}
$$

and $x_{1} \in R_{1}^{\prime}, x_{2} \in R_{1}^{\prime \prime}$. The morphism $h: \mathcal{C}_{p} \rightarrow \widetilde{X}_{0}$ contracts the chains $R^{\prime}, R^{\prime \prime}$. The pair $(\mathcal{E}, \varphi)$ is an admissible vector bundle $\mathcal{E}$ on $\mathcal{C}_{p}$ of rank $r$ and degree $d$ with an isomorphism $\varphi: \mathcal{E}_{y_{1}} \rightarrow \mathcal{E}_{y_{2}}$. Then, by definition,

$$
h_{\bullet}(\mathcal{E})=\left(h_{*} \mathcal{E}\left(-y_{1}-y_{2}\right)\right)\left(x_{1}+x_{2}\right):=\mathcal{F} .
$$


To see the GPB structure on $\mathcal{F}$, note that $R^{1} h_{*} \mathcal{E}\left(-y_{1}-y_{2}\right)=0$, we have

$$
0 \rightarrow h_{*} \mathcal{E}\left(-y_{1}-y_{2}\right) \rightarrow h_{*} \mathcal{E} \rightarrow\left({ }_{x_{1}} \mathcal{E}_{y_{1}}\right) \oplus\left({ }_{x_{2}} \mathcal{E}_{y_{2}}\right) \rightarrow 0
$$

where we use ${ }_{x} V$ to denote Skyscraper sheaf at point $x$ with fiber space $V$. The canonical morphism $h_{*}(\mathcal{E}) \rightarrow h_{*}(\mathcal{E})\left(x_{1}+x_{2}\right)$ induces a morphism

$$
h_{*}(\mathcal{E}) \rightarrow\left(h_{*} \mathcal{E}\left(-y_{1}-y_{2}\right)\right)\left(x_{1}+x_{2}\right)=\mathcal{F}
$$

thus a morphism $\left.\left({ }_{x_{1}} \mathcal{E}_{y_{1}}\right) \oplus\left({ }_{x_{2}} \mathcal{E}_{y_{2}}\right) \stackrel{\beta}{\rightarrow} \mathcal{F}\right|_{x_{1}+x_{2}}$ which gives the GPB structure

$$
\Gamma_{\varphi} \hookrightarrow \mathcal{E}_{y_{1}} \oplus \mathcal{E}_{y_{2}} \stackrel{\beta}{\longrightarrow} \mathcal{F}_{x_{1}} \oplus \mathcal{F}_{x_{2}}
$$

The kernel of morphism $\beta_{1}:=\left.\beta\right|_{\mathcal{E}_{y_{1}}}: \mathcal{E}_{y_{1}} \rightarrow \mathcal{F}_{x_{1}}$ (resp. $\beta_{2}: \mathcal{E}_{y_{2}} \rightarrow \mathcal{F}_{x_{2}}$ ) is the image of $H^{0}\left(\left.\mathcal{E}\right|_{R^{\prime}}\left(-x_{1}\right)\right) \rightarrow \mathcal{E}_{y_{1}}\left(\right.$ resp. $\left.H^{0}\left(\left.\mathcal{E}\right|_{R^{\prime \prime}}\left(-x_{2}\right)\right) \rightarrow \mathcal{E}_{y_{2}}\right)$ that send any section $s \in H^{0}\left(\left.\mathcal{E}\right|_{R^{\prime}}\left(-x_{1}\right)\right)$ to $s\left(y_{1}\right) \in \mathcal{E}_{y_{1}}$ (resp. $s \in H^{0}\left(\left.\mathcal{E}\right|_{R^{\prime \prime}}\left(-x_{2}\right)\right)$ to $\left.s\left(y_{2}\right) \in \mathcal{E}_{y_{2}}\right)$. Thus $\beta$ induces an injection $\beta: \Gamma_{\varphi} \hookrightarrow \mathcal{F}_{x_{1}} \oplus \mathcal{F}_{x_{2}}$. Now the image of $f$ at $p \in \mathbf{H}$ is

$$
f(p)=\left(\mathcal{F}, \beta\left(\Gamma_{\varphi}\right) \subset \mathcal{F}_{x_{1}} \oplus \mathcal{F}_{x_{2}}\right) \in \mathbf{G}
$$

Definition 2.7. $A G P B\left(\mathcal{F}, K \subset \mathcal{F}_{x_{1}} \oplus \mathcal{F}_{x_{2}}\right)$ is called $\alpha$-semistable (resp. $\alpha$-stable) if for any proper subbundle $\mathcal{F}^{\prime} \subset \mathcal{F}$ one has

$$
\chi\left(\mathcal{F}^{\prime}\right)-\alpha \operatorname{dim} \frac{\mathcal{F}_{x_{1}}^{\prime} \oplus \mathcal{F}_{x_{2}}^{\prime}}{K \cap\left(\mathcal{F}_{x_{1}}^{\prime} \oplus \mathcal{F}_{x_{2}}^{\prime}\right)} \leq(\text { resp. }<) \frac{\chi(\mathcal{F})-\alpha r}{r} r\left(\mathcal{F}^{\prime}\right)
$$

Proposition 2.8. The Gieseker vector bundle data $\left(\mathcal{C}_{p}, \mathcal{E}, \varphi\right)$ is $\alpha$-semistable (resp. $\alpha$-stable) if and only if the $G P B f(p)=\left(\mathcal{F}, \beta\left(\Gamma_{\varphi}\right) \subset \mathcal{F}_{x_{1}} \oplus \mathcal{F}_{x_{2}}\right)$ is $\alpha$-semistable (resp. $\alpha$-stable).

Proof. We prove firstly that $\alpha$-semistability of $\left(\mathcal{F}, \beta\left(\Gamma_{\varphi}\right) \subset \mathcal{F}_{x_{1}} \oplus \mathcal{F}_{x_{2}}\right)$ implies $\alpha$ semistability of $\left(\mathcal{C}_{p}, \mathcal{E}, \varphi\right)$. To recall the definition of $\beta: \mathcal{E}_{y_{1}} \oplus \mathcal{E}_{y_{2}} \rightarrow \mathcal{F}_{x_{1}} \oplus \mathcal{F}_{x_{2}}$ (see $[\mathrm{K} 2])$, consider the commutative diagram

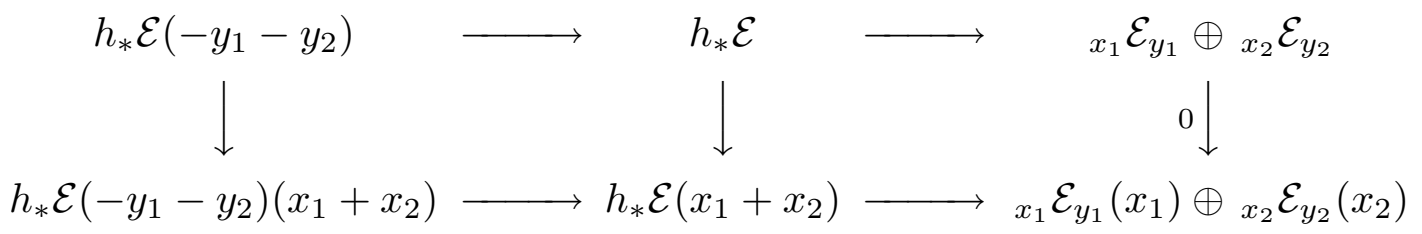

where the vertical maps are induced by the canonical map $\mathcal{O}_{\tilde{X}_{0}} \rightarrow \mathcal{O}_{\tilde{X}_{0}}\left(x_{1}+x_{2}\right)$. Thus the middle vertical map induces $\beta^{\prime}: h_{*} \mathcal{E} \rightarrow h_{*} \mathcal{E}\left(-y_{1}-y_{2}\right)\left(x_{1}+x_{2}\right):=\mathcal{F}$, which then induces $\beta: \mathcal{E}_{y_{1}} \oplus \mathcal{E}_{y_{2}} \rightarrow \mathcal{F}_{x_{1}} \oplus \mathcal{F}_{x_{2}}$ through the commutative diagram

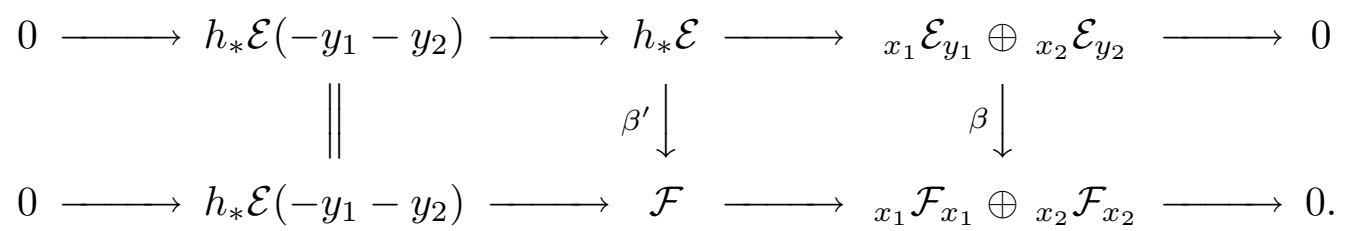


For any $\mathcal{E}^{\prime} \subset \mathcal{E}$ with $\mathcal{E} / \mathcal{E}^{\prime}$ torsion free, if $r_{0}\left(\mathcal{E}^{\prime}\right)=0$, it is clear that $\mathcal{E}^{\prime}$ satisfies the definition of $\alpha$-semistability since $\mathcal{E}$ is admissible. Thus we assume $r_{0}\left(\mathcal{E}^{\prime}\right)>0$. Let $\mathcal{F}^{\prime}=h_{*} \mathcal{E}^{\prime}\left(-y_{1}-y_{2}\right)\left(x_{1}+x_{2}\right)$, then we have

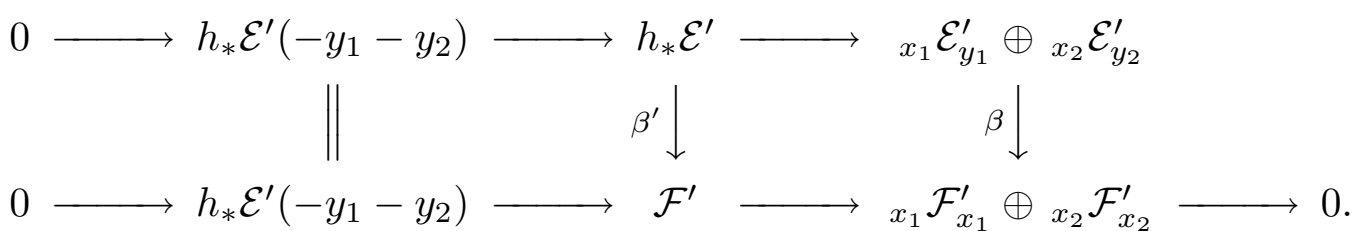

In particular, $\beta$ maps $\mathcal{E}_{y_{1}}^{\prime} \oplus \mathcal{E}_{y_{2}}^{\prime}$ to $\mathcal{F}_{x_{1}}^{\prime} \oplus \mathcal{F}_{x_{2}}^{\prime}$, thus we have

$$
\operatorname{dim} \Gamma_{\varphi} \cap\left(\mathcal{E}_{y_{1}}^{\prime} \oplus \mathcal{E}_{y_{2}}^{\prime}\right) \leq \operatorname{dim} \beta\left(\Gamma_{\varphi}\right) \cap\left(\mathcal{F}_{x_{1}}^{\prime} \oplus \mathcal{F}_{x_{2}}^{\prime}\right)
$$

Since $R^{1} h_{*} \mathcal{E}\left(-y_{1}-y_{2}\right)=0$, and $R^{1} h_{*} \mathcal{E}^{\prime}\left(-y_{1}-y_{2}\right)$ is a torsion sheaf, let $r^{\prime}$ be the rank of $\mathcal{F}^{\prime}$, we have

$$
\chi\left(\mathcal{E}^{\prime}\right) \leq \chi\left(\mathcal{F}^{\prime}\right)+\operatorname{dim}\left(\mathcal{E}_{y_{1}}^{\prime} \oplus \mathcal{E}_{y_{2}}^{\prime}\right)-2 r^{\prime} .
$$

By using (2.1) and (2.2), note $r_{0}\left(\mathcal{E}^{\prime}\right)=r^{\prime}$, we have

$$
\mu_{\alpha}\left(\mathcal{E}^{\prime}\right) \leq \frac{\chi\left(\mathcal{F}^{\prime}\right)-\alpha \operatorname{dim} \frac{\mathcal{F}_{x_{1}}^{\prime} \oplus \mathcal{F}_{x_{2}}^{\prime}}{\beta\left(\Gamma_{\varphi}\right) \cap\left(\mathcal{F}_{x_{1}}^{\prime} \oplus \mathcal{F}_{x_{2}}^{\prime}\right)}}{r^{\prime}}-2(1-\alpha) .
$$

When $\left(\mathcal{F}, \beta\left(\Gamma_{\varphi}\right) \subset \mathcal{F}_{x_{1}} \oplus \mathcal{F}_{x_{2}}\right)$ is $\alpha$-semistable (resp. $\alpha$-stable), we have

$$
\frac{\chi\left(\mathcal{F}^{\prime}\right)-\alpha \operatorname{dim} \frac{\mathcal{F}_{x_{1}}^{\prime} \oplus \mathcal{F}_{x_{2}}^{\prime}}{\beta\left(\Gamma_{\varphi}\right) \cap\left(\mathcal{F}_{x_{1}}^{\prime} \oplus \mathcal{F}_{x_{2}}^{\prime}\right)}}{r^{\prime}}-2(1-\alpha) \leq(<) \frac{\chi(\mathcal{F})-\alpha r}{r}-2(1-\alpha)=\mu_{\alpha}(\mathcal{E}) .
$$

Thus the Gieseker vector bundle data $\left(\mathcal{C}_{p}, \mathcal{E}, \varphi\right)$ is $\alpha$-semistable (resp. $\alpha$-stable).

To prove that $\alpha$-semistability of $\left(\mathcal{C}_{p}, \mathcal{E}, \varphi\right)$ implies $\alpha$-semistability of $\left(\mathcal{F}, \beta\left(\Gamma_{\varphi}\right) \subset\right.$ $\left.\mathcal{F}_{x_{1}} \oplus \mathcal{F}_{x_{2}}\right)$, we describe the map $\beta$ in detail. Let $T_{x_{1}} \subset \mathcal{E}_{x_{1}}$ (resp. $T_{x_{2}} \subset \mathcal{E}_{x_{2}}$ ) be the image of $H^{0}\left(\left.\mathcal{E}\right|_{R^{\prime}}\left(-y_{1}\right)\right) \rightarrow \mathcal{E}_{x_{1}}$ (resp. $\left.H^{0}\left(\left.\mathcal{E}\right|_{R^{\prime \prime}}\left(-y_{2}\right)\right) \rightarrow \mathcal{E}_{x_{2}}\right)$ that sends a section $s$ to its value $s\left(x_{1}\right)$ (resp. $s\left(x_{2}\right)$ ). Similarly, $T_{y_{1}} \subset \mathcal{E}_{y_{1}}\left(\right.$ resp. $T_{y_{2}} \subset \mathcal{E}_{y_{2}}$ ) is the image of $H^{0}\left(\mathcal{E}_{R^{\prime}}\left(-x_{1}\right)\right)$ (resp. $H^{0}\left(\mathcal{E}_{R^{\prime \prime}}\left(-x_{2}\right)\right)$ ). Let $\widetilde{\mathcal{E}}=\left.\mathcal{E}\right|_{\widetilde{X}_{0}}$, the canonical sequece $0 \rightarrow \mathcal{E}_{R^{\prime}}\left(-x_{1}-y_{1}\right) \oplus \mathcal{E}_{R^{\prime \prime}}\left(-x_{2}-y_{2}\right) \rightarrow \mathcal{E}\left(-y_{1}-y_{2}\right) \rightarrow \widetilde{\mathcal{E}} \rightarrow 0$ induces

$$
0 \rightarrow h_{*} \mathcal{E}\left(-y_{1}-y_{2}\right) \rightarrow \widetilde{\mathcal{E}} \rightarrow x_{1} \frac{\mathcal{E}_{x_{1}}}{T_{x_{1}}} \oplus x_{2} \frac{\mathcal{E}_{x_{2}}}{T_{x_{2}}} \rightarrow 0
$$

such that $h_{*} \mathcal{E}\left(-y_{1}-y_{2}\right)=\mathcal{F}\left(-x_{1}-x_{2}\right)$ is the Hecke modification of $\widetilde{\mathcal{E}}$ at $x_{1}$, $x_{2}$ along the subspaces $T_{x_{i}} \subset \mathcal{E}_{x_{i}}$ (note $\widetilde{\mathcal{E}}_{x_{i}}=\mathcal{E}_{x_{i}}$ ). In [KL], a homomorphism $\xi_{1}: \mathcal{E}_{y_{1}} \rightarrow \mathcal{E}_{x_{1}} / T_{x_{1}}$ (resp. $\xi_{2}: \mathcal{E}_{y_{2}} \rightarrow \mathcal{E}_{x_{2}} / T_{x_{2}}$ ) is introduced, which assigns to each $c \in \mathcal{E}_{y_{1}}\left(\operatorname{resp} . \quad c \in \mathcal{E}_{y_{2}}\right)$ the class $\left[s\left(x_{1}\right)\right] \in \mathcal{E}_{x_{1}} / T_{x_{1}}\left(\operatorname{resp} .\left[s\left(x_{2}\right)\right] \in \mathcal{E}_{x_{2}} / T_{x_{2}}\right)$ for some $s \in H^{0}\left(\mathcal{E}_{R^{\prime}}\right)$ (resp. $\left.s \in H^{0}\left(\mathcal{E}_{R^{\prime \prime}}\right)\right)$ such that $s\left(y_{1}\right)=c\left(\right.$ resp. $\left.s\left(y_{2}\right)=c\right)$. The homomorphism $\xi=\left(\xi_{1}, \xi_{2}\right): \mathcal{E}_{y_{1}} \oplus \mathcal{E}_{y_{2}} \rightarrow \frac{\mathcal{E}_{x_{1}}}{T_{x_{1}}} \oplus \frac{\mathcal{E}_{x_{2}}}{T_{x_{2}}}$ makes the diagram

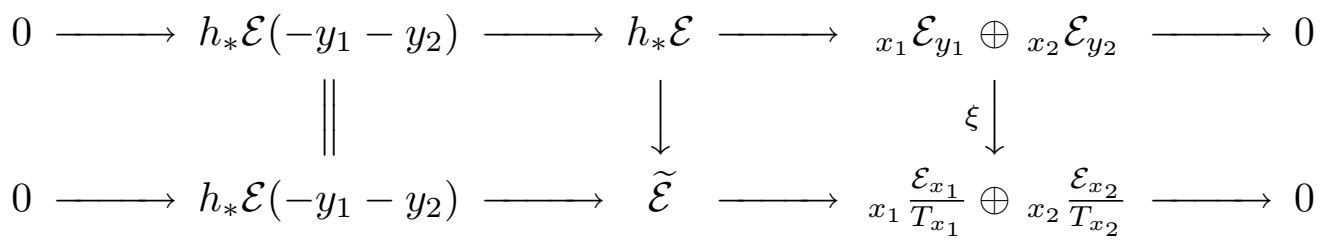


being commutative. Similarly, the canonical map $\widetilde{\mathcal{E}} \rightarrow \widetilde{\mathcal{E}}\left(x_{1}+x_{2}\right)$ induces an injection $\widetilde{\mathcal{E}} \stackrel{i}{\rightarrow} \mathcal{F}$, thus an injection ${ }_{x_{1}} \frac{\mathcal{E}_{x_{1}}}{T_{x_{1}}} \oplus \underset{x_{2}}{ } \frac{\mathcal{E}_{x_{2}}}{T_{x_{2}}} \stackrel{i}{\rightarrow}{ }_{x_{1}} \mathcal{F}_{x_{1}} \oplus{ }_{x_{2}} \mathcal{F}_{x_{2}}$ satisfying

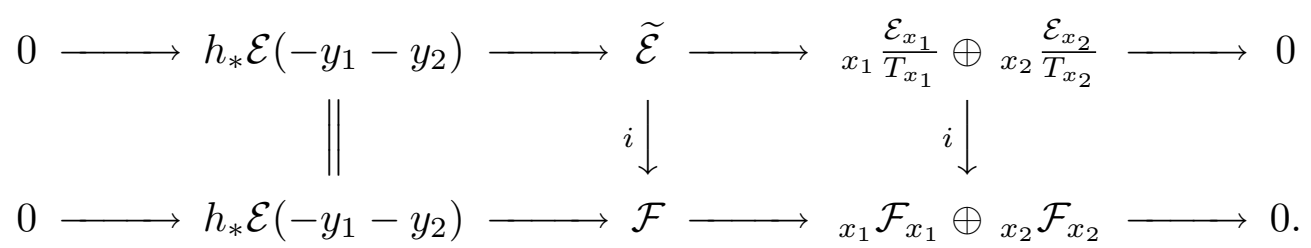

Then it is easy to see that $\beta^{\prime}: h_{*} \mathcal{E} \rightarrow \mathcal{F}$ (resp. $\beta: \mathcal{E}_{y_{1}} \oplus \mathcal{E}_{y_{2}} \rightarrow \mathcal{F}_{x_{1}} \oplus \mathcal{F}_{x_{2}}$ ) is the homomorphism $h_{*} \mathcal{E} \rightarrow \widetilde{\mathcal{E}} \stackrel{i}{\rightarrow} \mathcal{F}\left(\right.$ resp. $\left.\mathcal{E}_{y_{1}} \oplus \mathcal{E}_{y_{2}} \stackrel{\xi}{\rightarrow} \frac{\mathcal{E}_{x_{1}}}{T_{x_{1}}} \oplus \frac{\mathcal{E}_{x_{2}}}{T_{x_{2}}} \stackrel{i}{\rightarrow} \mathcal{F}_{x_{1}} \oplus \mathcal{F}_{x_{2}}\right)$.

For any proper subbundle $\mathcal{F}^{\prime} \subset \mathcal{F}$ of rank $r^{\prime}$, let $\widetilde{\mathcal{E}}^{\prime}=k e r\left\{\widetilde{\mathcal{E}} \stackrel{i}{\rightarrow} \mathcal{F} \rightarrow \mathcal{F} / \mathcal{F}^{\prime}\right\}$ (which is a subbundle of $\widetilde{\mathcal{E}}$ since $\mathcal{F} / \mathcal{F}^{\prime}$ is a bundle). We still use $\widetilde{\mathcal{E}}^{\prime} x_{i} \subset \mathcal{E}_{x_{i}}$ to denote the image of $\widetilde{\mathcal{E}}^{\prime}{ }_{x_{i}} \subset \widetilde{\mathcal{E}}_{x_{i}}$ under the identification $\widetilde{\mathcal{E}}_{x_{i}}=\mathcal{E}_{x_{i}}$. Let $B \subset \Gamma_{\varphi}$ be the subspace such that $\beta(B)=\beta\left(\Gamma_{\varphi}\right) \cap\left(\mathcal{F}_{x_{1}}^{\prime} \oplus \mathcal{F}_{x_{2}}^{\prime}\right)$ and $B_{i} \subset \mathcal{E}_{y_{i}}$ be projections of $B$. Then it is easy to check that $\xi_{i}\left(B_{i}\right) \subset \sigma_{i}\left(\widetilde{\mathcal{E}}^{\prime} x_{i}\right)$ under the canonical projection $\mathcal{E}_{x_{i}} \stackrel{\sigma_{i}}{\longrightarrow} \mathcal{E}_{x_{i}} / T_{x_{i}}$. By Lemma 2.2 of [KL], there is a subsheaf $\mathcal{E}_{1}^{\prime} \subset \mathcal{E}_{R^{\prime}}$ (resp. $\left.\mathcal{E}_{2}^{\prime} \subset \mathcal{E}_{R^{\prime \prime}}\right)$ such that $\operatorname{Im}\left\{\left.\left.\mathcal{E}_{1}^{\prime}\right|_{x_{1}} \rightarrow \mathcal{E}_{R^{\prime}}\right|_{x_{1}}\right\}=\widetilde{\mathcal{E}}_{x_{1}}, \operatorname{Im}\left\{\left.\left.\mathcal{E}_{1}^{\prime}\right|_{y_{1}} \rightarrow \mathcal{E}_{R^{\prime}}\right|_{y_{1}}\right\}=B_{1}$ (resp. $\operatorname{Im}\left\{\left.\left.\mathcal{E}_{2}^{\prime}\right|_{x_{2}} \rightarrow \mathcal{E}_{R^{\prime \prime}}\right|_{x_{2}}\right\}=\widetilde{\mathcal{E}}_{x_{2}}^{\prime}, \operatorname{Im}\left\{\left.\left.\mathcal{E}_{2}^{\prime}\right|_{y_{2}} \rightarrow \mathcal{E}_{R^{\prime \prime}}\right|_{y_{2}}\right\}=B_{2}$ ) and $\chi\left(\mathcal{E}_{i}^{\prime}\right) \geq$ $\operatorname{dim}\left(\widetilde{\mathcal{E}}^{\prime}{ }_{x_{i}}\right)+\operatorname{dim}\left(B_{i}\right)-\operatorname{dim} \sigma_{i}\left(\widetilde{\mathcal{E}}^{\prime}{ }_{x_{i}}\right)$. Glue the subsheaves $\left.\widetilde{\mathcal{E}}^{\prime} \subset \mathcal{E}\right|_{\widetilde{X}_{0}}, \mathcal{E}_{1}^{\prime} \subset \mathcal{E}_{R^{\prime}}, \mathcal{E}_{2}^{\prime} \subset$ $\mathcal{E}_{R^{\prime \prime}}$ together, one get a subsheaf $\mathcal{E}^{\prime} \subset \mathcal{E}$ such that $r_{0}\left(\mathcal{E}^{\prime}\right)=r k\left(\mathcal{F}^{\prime}\right)=r^{\prime}$ and

$$
\operatorname{Im}\left\{\mathcal{E}_{y_{1}}^{\prime} \oplus \mathcal{E}_{y_{2}}^{\prime} \rightarrow \mathcal{E}_{y_{1}} \oplus \mathcal{E}_{y_{2}}\right\}=B_{1} \oplus B_{2}, \quad \chi\left(\mathcal{E}^{\prime}\right)=\chi\left(\widetilde{\mathcal{E}}^{\prime}\right)+\chi\left(\mathcal{E}_{1}^{\prime}\right)+\chi\left(\mathcal{E}_{2}^{\prime}\right)-2 r^{\prime}
$$

Note $\chi\left(\widetilde{\mathcal{E}}^{\prime}\right)-\operatorname{dim} \sigma_{1}\left(\widetilde{\mathcal{E}}_{x_{1}}\right)-\operatorname{dim} \sigma_{2}\left(\widetilde{\mathcal{E}}^{\prime}{ }_{x_{2}}\right)=\chi\left(\mathcal{F}^{\prime}\right)-2 r^{\prime}$, we have

$$
\chi\left(\mathcal{E}^{\prime}\right) \geq \chi\left(\mathcal{F}^{\prime}\right)-2 r^{\prime}+\operatorname{dim}\left(B_{1} \oplus B_{2}\right) .
$$

Using $(2.3)$ and $\beta(B)=\beta\left(\Gamma_{\varphi}\right) \cap\left(\mathcal{F}_{x_{1}}^{\prime} \oplus \mathcal{F}_{x_{2}}^{\prime}\right)$, one has

$$
\frac{\chi\left(\mathcal{F}^{\prime}\right)-\alpha \operatorname{dim} \frac{\mathcal{F}_{x_{1}}^{\prime} \oplus \mathcal{F}_{x_{2}}^{\prime}}{\beta\left(\Gamma_{\varphi}\right) \cap\left(\mathcal{F}_{x_{1}}^{\prime} \oplus \mathcal{F}_{x_{2}}^{\prime}\right)}}{r^{\prime}} \leq \mu_{\alpha}\left(\mathcal{E}^{\prime}\right)+2(1-\alpha) .
$$

Then $\mu_{\alpha}\left(\mathcal{E}^{\prime}\right) \leq \mu_{\alpha}(\mathcal{E})$ (resp. $\left.\mu_{\alpha}\left(\mathcal{E}^{\prime}\right)<\mu_{\alpha}(\mathcal{E})\right)$ implies

$$
\mu_{\alpha}\left(\mathcal{E}^{\prime}\right)+2(1-\alpha) \leq(\text { resp. }<) \frac{\chi(\mathcal{F})-\alpha r}{r} .
$$

Thus $\alpha$-semistability (resp. $\alpha$-stability) of $\left(\mathcal{C}_{p}, \mathcal{E}, \varphi\right)$ implies $\alpha$-semistability (resp. $\alpha$-stability) of $\left(\mathcal{F}, \beta\left(\Gamma_{\varphi}\right) \subset \mathcal{F}_{x_{1}} \oplus \mathcal{F}_{x_{2}}\right)$.

The action of $P G L(\widetilde{P}(N))$ on the Quot scheme induces actions on $\mathbf{H}$ and $\mathbf{G}$ such that $f: \mathbf{H} \rightarrow \mathbf{G}$ is a $P G L(\widetilde{P}(N))$-equivariant morphism. Let $\widetilde{\mathbf{Q}}$ be the closure of $\mathbf{Q}$ in the Quot scheme with universal quotient $\widetilde{\mathcal{F}}$ on $\widetilde{X}_{0} \times \widetilde{\mathbf{Q}}$. Let $\widetilde{\mathbf{G}} \subset$ $\operatorname{Grass}_{r}\left(\widetilde{\mathcal{F}}_{x_{1}} \oplus \widetilde{\mathcal{F}}_{x_{2}}\right)$ be the closure of $\mathbf{G}$ and $\widetilde{\mathbf{H}}$ be the closure of $\mathbf{H}$ in $\widetilde{\mathbf{P}}$, where $\widetilde{\mathbf{P}}$ is obtained by performing blowing ups of $\mathbb{P}\left(\left(\mathcal{H o m}\left(\widetilde{\mathcal{F}}_{x_{1}}, \widetilde{\mathcal{F}}_{x_{2}}\right) \oplus \mathcal{O}_{\widetilde{\mathbf{Q}}}\right)^{\vee}\right)$. Then it is clear that $\widetilde{\mathbf{H}}$ and $\widetilde{\mathbf{G}}$ have the induced $P G L(\widetilde{P}(N))$-action. Let $\mathbf{W} \subset \widetilde{\mathbf{H}} \times \widetilde{\mathbf{G}}$ 
be the graph of the rational map $f: \widetilde{\mathbf{H}} \rightarrow \widetilde{\mathbf{G}}$ and $p: \mathbf{W} \rightarrow \widetilde{\mathbf{G}}$ the projection, which is a $P G L(\widetilde{P}(N))$-equivariant morphism and $\left.p\right|_{\mathbf{H}}=f$. Thus we have the $P G L(\widetilde{P}(N))$-equivariant factorization

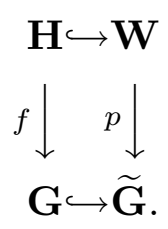

According to [B], there exist a family of polarizations $L_{\alpha}(0<\alpha<1)$ on $\widetilde{\mathbf{G}}$ such that GIT stability (resp. semistability) for $L_{\alpha}$ is equivalent to $\alpha$-stability (resp. $\alpha$-semistability) of the corresponding GPB. Fix a polarization $\mathcal{O}_{\mathbf{W}}(1)$ and consider the polarization $\mathcal{L}_{\alpha}=\mathcal{O}_{\mathbf{W}}(1) \otimes p^{*}\left(L_{\alpha}^{m}\right)$ on $\mathbf{W}$. Then, when $m$ is sufficiently large, we have the following relationships of GIT stable (resp. semistable) locus

$$
p^{-1}\left(\widetilde{\mathbf{G}}\left(L_{\alpha}\right)^{s}\right) \subset \mathbf{W}\left(\mathcal{L}_{\alpha}\right)^{s} \subset \mathbf{W}\left(\mathcal{L}_{\alpha}\right)^{s s} \subset p^{-1}\left(\widetilde{\mathbf{G}}\left(L_{\alpha}\right)^{s s}\right)
$$

by the general facts of GIT. Let $\mathbf{G}_{\alpha}^{s}$ (resp. $\mathbf{G}_{\alpha}^{s s}$ ) be the open set of $\mathbf{G}$ consisting of $\alpha$-stable (resp. $\alpha$-semistable) GPB, and let $\mathbf{H}_{\alpha}^{s}\left(\operatorname{resp} . \mathbf{H}_{\alpha}^{s s}\right)$ be the open set of $\mathbf{H}$ consisting of $\alpha$-stable (resp. $\alpha$-semistable) Gieseker vector bundle data. Then, by the results of $[\mathrm{B}]$, we have $\widetilde{\mathbf{G}}\left(L_{\alpha}\right)^{s}=\mathbf{G}_{\alpha}^{s}$ and $\widetilde{\mathbf{G}}\left(L_{\alpha}\right)^{s s}=\mathbf{G}_{\alpha}^{s s}$. Since $f$ is proper, we have $p^{-1}\left(\mathbf{G}_{\alpha}^{s}\right)=f^{-1}\left(\mathbf{G}_{\alpha}^{s}\right), p^{-1}\left(\mathbf{G}_{\alpha}^{s s}\right)=f^{-1}\left(\mathbf{G}_{\alpha}^{s s}\right)$. By Proposition 2.8, we have

Lemma 2.9. For any $0<\alpha<1$, when $m$ is large enough, we have

$$
\mathbf{H}_{\alpha}^{s} \subset \mathbf{W}\left(\mathcal{L}_{\alpha}\right)^{s} \subset \mathbf{W}\left(\mathcal{L}_{\alpha}\right)^{s s} \subset \mathbf{H}_{\alpha}^{s s}
$$

When $\alpha \notin I_{r}$ (the set of critical values), we have

$$
\mathbf{H}_{\alpha}^{s}=\mathbf{W}\left(\mathcal{L}_{\alpha}\right)^{s}=\mathbf{W}\left(\mathcal{L}_{\alpha}\right)^{s s}=\mathbf{H}_{\alpha}^{s s}
$$

Theorem 2.10. Let $M^{\alpha}:=\mathbf{W}\left(\mathcal{L}_{\alpha}\right)^{s s} / / P G L(\widetilde{P}(N))$ be the GIT quotient. Then, when $\alpha \notin I_{r}, M^{\alpha}$ is the fine moduli space of $\alpha$-stable Gieseker vector bundle data.

Proof. It is not difficult to check that two points of $\mathbf{H}$ are in the same orbit if and only if the corresponding Gieseker vector bundle data are equivalent. When $\alpha \notin I_{r}$, by Lemma 2.9, $M^{\alpha}$ is a smooth projective variety whose closed points are precisely the equivalent classes of $\alpha$-stable Gieseker vector bundle data.

To show the existence of a universal object, we show the universal generalized isomorphism from $\mathcal{F}_{x_{1}}$ to $\mathcal{F}_{x_{2}}$ on $\mathbf{H}_{\alpha}^{s}=W\left(\mathcal{L}_{\alpha}\right)^{s}$ descends to $M^{\alpha}$, where $\mathcal{F}$ is the pullback of the universal quotient. From the construction of universal generalized isomorphism (see [K1]), it is enough to show the bundles $\mathcal{F}_{x_{i}}$ and the divisors $Z_{i}$, $Y_{i}$ descend to $M^{\alpha}$. To see this, note firstly the stabilizer of any point of $\mathbf{H}_{\alpha}^{s}$ consists of scales $\lambda \cdot I \subset G L(\widetilde{P}(N))$, and $\mathbf{H}=K G L\left(\mathcal{F}_{x_{1}}, \mathcal{F}_{x_{2}}\right)$ is unchanged if we tensor $\mathcal{F}$ by a line bundle on the Quot scheme. Then, by tensor $\mathcal{F}$ with a line bundle on the Quot scheme, we can make any scale acting on $\mathcal{F}$ trivially since $(\chi, r)=1$. Thus there is a universal generalized isomorphism on $\mathbf{H}_{\alpha}^{s}$ that descends to $M^{\alpha}$, which gives a universal Gieseker vector bundle data on $M^{\alpha}$ by the construction of [K2]. 


\section{REFERENCES}

[B] Bhosle, U. N., Generalized parabolic bundles and applications II, Proc. Indian Acad. Sci.(Math. Sci.) 106 (1996), 403-420.

[BK] M. Brion and I. Kausz, Vanishing of top equivariant Chern classes of regular embeddings, math.AG/0503196 (2005).

[Gi] D. Gieseker, A degeneration of the moduli space of stable bundles, J. Differential Geom. 19 (1984), 173-206.

[K1] Ivan Kausz, A modular compactification of the general linear group, Doc. Math. 5 (2000), 553-594.

[K2] Ivan Kausz, A Gieseker type degeneration of moduli stacks of vector bundles on curves, math.AG/0201197 (2002), 1-59.

[KL] Young-Hoon Kiem and Jun Li, Vanishing of the top Chern classes of the moduli of vector bundles, math.AG/0403033 (2004).

[NS] D.S. Nagaraj and C.S. Seshadri, Degenerations of the moduli spaces of vector bundles on curves II. Generalized Gieseker moduli spaces, Proc. Indian Acad. Sci.(Math. Sci.) 109 (1999), 165-201.

[Tei] M. Teixidor, Brill-Noether theory for stable vector bundles, Duke Math. J. 62 (1991), 385-400.

Institute of Mathematics, Chinese Academy of Sciences, Beijing 100080, China

E-mail address: xsun@math.ac.cn 